\title{
Elevated secondary infection rates in patients with coronavirus disease 2019 (COVID-19) requiring extracorporeal membrane oxygenation
}

\author{
Joseph E. Marcus MD ${ }^{1}$ (1), Valerie G. Sams MD $^{2}$ and Alice E. Barsoumian MD ${ }^{1}$ \\ ${ }^{1}$ Infectious Disease Service, Department of Internal Medicine, Joint Base San Antonio Fort Sam Houston, San Antonio, Texas and ${ }^{2}$ Department of Surgery, \\ Brooke Army Medical Center, Joint Base San Antonio Fort Sam Houston, San Antonio Texas
}

Coronavirus disease 2019 (COVID-19) has had a tremendous impact in healthcare, including a surge of hospitalizations. To prevent inhospital outbreaks, contact and airborne precautions have been implemented for patients with COVID-19 with demonstrated success in preventing severe acute respiratory coronavirus virus 2 (SARS-CoV-2) transmission. ${ }^{1}$ Data on the rates of secondary infections in patients hospitalized with COVID-19 are limited. ${ }^{2}$ Although early studies showed low rates of secondary infections in patients with COVID-19, ${ }^{3}$ more recent studies in both larger and sicker populations have shown elevated rates of secondary infection. ${ }^{4-6}$

Extracorporeal membrane oxygenation (ECMO) is used for pulmonary bypass in patients with reversible respiratory failure, and it poses a significant risk of secondary infections. ${ }^{7}$ ECMO is currently only recommended for COVID-19 patients with few comorbidities and without severe multisystem organ failure. ${ }^{8}$ Although it has been utilized worldwide for patients with COVID-19, no data on secondary infections in these patients are available. ${ }^{9}$ In this study, we retrospectively compared secondary infection rates on ECMO for patients with COVID-19 to patients with influenza. Although both viruses can cause devastating pulmonary disease, unlike influenza, patients hospitalized with COVID-19 are treated with immunosuppression. Additionally, COVID-19 has been associated with high patient volumes, which strained healthcare systems. As such, we hypothesized that there would be more secondary infections with COVID-19, despite the increased use of PPE and emphasis on infection prevention.

\section{Methods}

All patients who completed a course of ECMO at Brooke Army Medical Center between January 1, 2013, and October 10, 2020, with confirmed influenza or severe acute respiratory coronavirus virus 2 (SARS-CoV-2) were included in this retrospective analysis. Positive cultures during ECMO course or within 48 hours of decannulation that were determined to be pathogenic by the patient's treatment team were labeled as bloodstream, respiratory, or urinary infections based on the site of culture. Culture organisms that were considered

\footnotetext{
Author for correspondence: Joseph E Marcus, E-mail: joseph.e.marcus3.mil@mail.mil Cite this article: Marcus JE, Sams VG, and Barsoumian AE. (2021). Elevated secondary infection rates in patients with coronavirus disease 2019 (COVID-19) requiring extracorporeal membrane oxygenation. Infection Control \& Hospital Epidemiology, 42: 770-772, https://doi.org/10.1017/ice.2021.61
}

colonizers or contaminants by the treatment team were excluded. Multidrug-resistant organisms (MDROs) were defined as resistance to 3 or more classes of antibiotics.

We compared patients with influenza and COVID-19 by demographics, duration of hospitalization prior to ECMO cannulation, length of stay, mortality, number of infections, infection rates per 1,000 ECMO patient days, and MDRO rate. Nominal variables and rates were compared using the $\chi^{2}$ or Fisher exact test as appropriate, whereas continuous variables were compared by Mann-Whitney $U$ test. A $P$ value of .05 was considered significant.

\section{Results}

Of the 210 patients who received ECMO during the study period, 39 patients (19\%) were diagnosed with either COVID-19 or influenza. All patients received the venovenous modality of ECMO. Overall, 4 patients (10\%) who completed their ECMO course were still inpatients and 35 patients (90\%) had completed their hospital course as of October 10,2020, with a survival rate to hospital discharge of $72 \%$.

We detected minimal differences in the demographics of patients who underwent ECMO with influenza versus COVID-19 (Table 1). All patients with COVID-19 were treated with immunosuppression during their hospital course. Patients with COVID-19 were hospitalized longer prior to ECMO cannulation than patients with influenza (median 12 [IQR, 8-14] days vs 5 [IQR, 3-8]; $P=.001$ ).

For the primary outcome, patients with COVID-19 had greater rates of secondary infection while on ECMO (37.3 per 1,000 patient days vs $17.7 ; P=.04)$. Infections occurred earlier after cannulation in patients with COVID-19 (median day 5 [IQR, 3-8] vs 16 [IQR, $10-21] ; P=.03$ ). However, there was no difference in day of infection after hospital admission (19 [IQR, 14-26] vs 21 [IQR, 16-25]; $P=.92)$. MDROs were isolated at similar frequencies in the 2 groups ( $17 \%$ vs $36 \% ; P=.60)$.

\section{Discussion}

In this study, we compared patients with respiratory viruses requiring ECMO, and we detected an elevated secondary infection rate for patients with COVID-19. The reasons for this difference are likely multifactorial and include strain on the healthcare system, the use of immunosuppressants, and possible COVID-19 
Table 1. Characteristics of Patients Receiving Extracorporeal Membrane Oxygenation (ECMO) With Influenza or COVID-19

\begin{tabular}{|c|c|c|c|c|}
\hline Characteristic & All & Influenza $(n=22)$ & COVID-19 $(n=17)$ & $P$ Value $^{\mathrm{a}}$ \\
\hline Age, median y (IQR) & $43(34-53)$ & $45(32-55)$ & $42(35-49)$ & .76 \\
\hline Sex, male, no. (\%) & $28(70)$ & $15(68)$ & $13(76)$ & .72 \\
\hline \multicolumn{5}{|l|}{ Comorbidities, no. (\%) } \\
\hline Obesity & $25(63)$ & $15(68)$ & $10(58)$ & .51 \\
\hline Hypertension & $13(33)$ & $8(36)$ & $5(29)$ & .74 \\
\hline Diabetes mellitus & $11(28)$ & $6(27)$ & $5(29)$ & 1 \\
\hline Days in hospital before ECMO, median d (IQR) & $8(4-13)$ & $5(3-8)$ & $12(8-14)$ & .001 \\
\hline Hours on ECMO, median h (IQR) & $360(200-610)$ & $360(196-604)$ & $321(191-441)$ & .93 \\
\hline Days of hospitalization, median d (IQR) & $38(27-47)^{b}$ & $36(28-43)$ & $38(28-54)^{b}$ & .43 \\
\hline Survival to discharge, no. (\%) & $26(67)^{b}$ & $17(89)$ & $9(69)^{b}$ & .46 \\
\hline Days to first ECMO infection after cannulation, median d (IQR) & $8(5-16)$ & $18(10-21)$ & $5(3-7)$ & .01 \\
\hline Days to first ECMO infection after hospitalization, median d (IQR) & $20(14-26)$ & $21(16-25)$ & $19(14-26)$ & .92 \\
\hline \multicolumn{5}{|l|}{ Patients with infection, no. (\%) } \\
\hline Any infection & $18(46)$ & $8(36)$ & $10(58)$ & .22 \\
\hline BSI & $13(33)$ & $6(27)$ & $7(41)$ & .60 \\
\hline RI & $7(18)$ & $2(9)$ & $5(29)$ & .21 \\
\hline UTI & $1(3)$ & $1(5)$ & 0 & \\
\hline \multicolumn{5}{|l|}{ Infections per 1,000 ECMO days } \\
\hline Total & 27.2 & 17.7 & 37.3 & .04 \\
\hline BSI & 16.8 & 13.2 & 21.8 & .31 \\
\hline $\mathrm{RI}$ & 9.1 & 4.4 & 15.6 & .19 \\
\hline UTI & 1.3 & 2.2 & 0 & .79 \\
\hline Multidrug-resistant bacteria & $5 / 17(29)$ & $1 / 6(17)$ & $4 / 11(36)$ & .6 \\
\hline
\end{tabular}

Note. IQR, interquartile range; BSI, bloodstream infection; RI, respiratory infection, UTI, urinary tract infection.

apresented as no. (\%) or median (IQR).

${ }^{b} \chi^{2}$, Fisher exact, or Wilcoxon rank-sum test.

'Excludes 4 patients with COVID-19 who were still inpatients as of October 10, 2020.

disease-specific characteristics. Overall, the rate of infections of 37.1 per 1,000 patient days in patients with COVID-19 is higher than the national average for all adults who receive ECMO of 30.6. ${ }^{7}$

Infections tended to occur earlier in the ECMO course for patients with COVID-19 than for patients with influenza. Despite similar time on ECMO circuit between the 2 groups, no patient with COVID-19 had an infection after ECMO day 17, whereas 5 infections occurred after that day in patients with influenza. Additionally, patients with COVID-19 had longer hospital courses pre-ECMO, which may contribute to the timing of infection onset. Further studies are needed to evaluate the risk factors contributing to secondary infection.

This study has several limitations. It was a retrospective, singlecenter study with a small number of patients, and it may be underpowered to detect differences in specific types of infections and MDROs. Secondly, COVID-19 and influenza have different pathophysiology and comparisons may be premature. We were not able to differentiate whether secondary infections are caused by a failure of infection prevention practices, overall strain on the healthcare system, or due to differences in the underlying disease process. Finally, we have no data on adherence to PPE and hand hygiene.

In this study, we compared critically ill patients that presented with similar demographics to an established ECMO center with adequate resources throughout the pandemic. Our results show that the risk of secondary infections is significant for this population.
Infection control strategies should continue to be implemented that protect healthcare workers, with emphasis on adherence to infection prevention and control bundles. However, secondary infections for COVID-19 patients on ECMO may persist due to unrecognized factors. Larger, multicenter trials with COVID-19 patients are needed to determine the best practices for caring for these patients to reduce secondary infections.

Acknowledgments. The view(s) expressed herein are those of the author(s) and do not reflect the official policy or position of Brooke Army Medical Center, the US Air Force Medical Department, the US Air Force Office of the Surgeon General, the Department of the Army, the Department of the Air Force and Department of Defense, or the US government.

Financial support. No financial support was provided relevant to this article.

Conflicts of interest. All authors report no conflicts of interest relevant to this article.

\section{References}

1. Lepak AJ, Shirley DK, Buys A, Stevens L, Safdar N. Implementation of infection control measures to prevent healthcare-associated transmission of SARS-CoV-2. Infect Control Hosp Epidemiol 2020. doi: 10.1017/ice.2020.1262.

2. Zhou P, Liu Z, Chen Y, Xiao Y, Huang X, Fan XG. Bacterial and fungal infections in COVID-19 patients: a matter of concern. Infect Control Hosp Epidemiol 2020;41:1124-1125. 
3. Nori $\mathrm{P}$, Cowman $\mathrm{K}$, Chen V, et al. Bacterial and fungal coinfections in COVID-19 patients hospitalized during the New York City pandemic surge. Infect Control Hosp Epidemiol 2020;42:84-88.

4. Giacobbe DR, Battaglini D, Ball L, et al. Bloodstream infections in critically ill patients with COVID-19. Eur J Clin Investig 2020;50:e13319.

5. Cates J, Lucero-Obusan C, Dahl RM, et al. Risk for in-hospital complications associated with COVID-19 and influenza-Veterans' Health Administration, United States, October 1, 2018-May 31, 2020. Morbid Mortal Wkly Rep 2020.

6. Ripa M, Galli L, Poli A, et al. Secondary infections in patients hospitalized with COVID-19: incidence and predictive factors. Clin Microbiol Infect $2020 ; 69 ; 1528-1534$
7. Bizzarro MJ, Conrad SA, Kaufman DA, Rycus P. Infections acquired during extracorporeal membrane oxygenation in neonates, children, and adults. Pediatr Crit Care Med 2011;12:277-281.

8. Shekar K, Badulak J, Peek G, et al. Extracorporeal Life Support Organization coronavirus disease 2019 interim guidelines: a consensus document from an international group of interdisciplinary extracorporeal membrane oxygenation providers. Am Soc Artif Intern Organs J 2020;66:707-721.

9. Barbaro RP, MacLaren G, Boonstra PS, et al. Extracorporeal membrane oxygenation support in COVID-19: an international cohort study of the Extracorporeal Life Support Organization registry. Lancet 2020;396: 1071-1078.

\title{
Predictors for in-hospital mortality from coronavirus disease 2019 (COVID-19) infection among adults aged 18-65 years
}

\author{
Ashish Bhargava MD, FACP (1), Mamta Sharma MD, Elisa Akagi MD, Susanna M. Szpunar PhD and \\ Louis Saravolatz MD, MACP, FIDSA
}

Ascension St John Hospital, Detroit, Michigan

As of October 13,2020, at least 37,867,739 cases of coronavirus disease 2019 (COVID-19) had been identified worldwide, including $\sim 7,804,699$ cases in the United States. ${ }^{1}$ Early data from the United States showed that $20 \%$ of deaths occurred in patients aged $20-64$ years and $80 \%$ occurred in patients aged $\geq 65$ years. $^{2}$ Understanding the risk factors associated with mortality among adults aged $<65$ years may identify vulnerable patients. The purpose of the study was to identify factors present at the time of hospital admission that predicted in-hospital mortality from COVID-19 among adults aged $\leq 65$ years.

\section{Methods}

We conducted a retrospective study at a 776-bed, tertiary-care center. The study was approved by the Ascension St John Hospital Institutional Review Board. We included adult patients with confirmed COVID-19 by positive real-time reverse-transcriptase-polymerase chain reaction (RT-PCR) assay of a nasopharyngeal swab for severe acute respiratory coronavirus virus 2 (SARS-CoV-2) from March 8 to June 14, 2020. Patient demographics, comorbid conditions, presenting symptoms, initial vital signs, admission laboratory and radiological findings, and outcome variables were extracted from the electronic medical records.

Statistical analyses were performed using SPSS version 27.0 software (IBM, Armonk, NY). Univariable analyses were conducted using the Student $t$ test, the Mann-Whitney U test, and $\chi^{2}$ analysis. Variables that were significant or near-significant predictors of mortality $(P<.09)$ were entered into a multivariable logistic regression model using a forward likelihood ratio algorithm. For comorbidities, the Charlson weighted index of comorbidity

Author for correspondence: Ashish Bhargava, E-mail: Ashish.bhargava@ascension. org. Or Louis Saravolatz, E-mail: louis.saravolatz@ascension.org

Cite this article: Bhargava A, et al. (2021). Predictors for in-hospital mortality from coronavirus disease 2019 (COVID-19) infection among adults aged 18-65 years. Infection Control \& Hospital Epidemiology, 42: 772-775, https://doi.org/10.1017/ ice. 2020.1390
(CWIC) score was used instead of individual comorbid conditions. ${ }^{3}$ Results from the regression are reported as odds ratios with $95 \%$ confidence intervals. All reported $P$ values are 2 -sided.

\section{Results}

In total, 265 hospitalized patients, ages 18-65 years, were included in this study. The mean age of the cohort was 50.4 years (SD, 10.7), 140 (52.8\%) were male, and $226(85.3 \%)$ were black/AfricanAmerican. The mean body mass index of the cohort was 35.8 $\mathrm{kg} / \mathrm{m}^{2}$ (SD, 9.3). The mean duration of symptoms prior to hospitalization was 5.8 days (SD, 3.8). Severe pneumonia was diagnosed in 49 patients (18.5\%). Mechanical ventilation was required for 66 patients (24.9\%). Overall, 214 patients $(80.8 \%)$ improved clinically and survived to discharge.

The in-hospital case fatality rate (CFR) was $19.2 \%$ (51 of 265). Patients who died were significantly older (mean age, 53.4 years [SD, 9.4] vs 49.7 years [SD, 10.8]) than patients who survived $(P=.03)$. Patients who died were significantly more likely to have hypertension, diabetes with chronic complications, hemiplegia, preexisting renal disease, liver disease, and a diagnosis of malignancy (Table 1). The prevalence of lymphocytopenia and thrombocytopenia were higher among patients who died than those who survived.

The Quick Sequential Organ Failure Assessment (qSOFA) score $(0-3)$ is composed of 3 clinical parameters with 1 point allotted to each of these parameters: systolic blood pressure $\leq 100 \mathrm{~mm} \mathrm{Hg}$, respiratory rate $\geq 22$ breaths per minute, and altered mental status. For patients with qSOFA scores of $0,1,2$, and 3 at the time of hospital admission, the mortality rates were $9.7 \%, 21.1 \%, 41.7 \%$, and $60 \%$, respectively $(P<.0001)$.

The final multivariable logistic regression model included 4 variables that predicted increased odds of death in patients with COVID-19 infection: Charlson score, presence of hypertension, qSOFA, and thrombocytopenia at the time of hospital admission (Table 2).

(c) The Author(s), 2020. Published by Cambridge University Press on behalf of The Society for Healthcare Epidemiology of America. This is an Open Access article, distributed under the terms of the Creative Commons Attribution licence (http://creativecommons.org/licenses/by/4.0/), which permits unrestricted re-use, distribution, and reproduction in any medium, provided the original work is properly cited. 